\title{
Techniques and Applications of Face Recognition : An Overview
}

${ }^{1}$ Dr. Bharti Kukreja, ${ }^{2}$ Ritul Kumar Sharma, ${ }^{2}$ Shubham Sinha

${ }^{1}$ Associate Professor, B.S Anangpuria institute of Technology and Management, Faridabad, Haryana, India

${ }^{2}$ Research Scholar, B.S Anangpuria institute of Technology and Management, Faridabad, Haryana, India

\section{ABSTRACT}

Face recognition is one of the most used technologies for security and privacy in electronic gadgets, automotive industry and other domains as well. It is also the most reliable form of biometric authentications. In the present paper the basic process of Face Recognition technology, biological aspects behind the smooth functioning of face recognition, conditions affecting the process (consisting of twins face, hairstyle change and time-to-time face change due to ageing) have been explained. Effort has also been made to enumerate the uses of face recognition in different technological approaches and the huge market value for this emerging biometric solution.

Keywords :Face Recognition, Authentication, Security, Privacy.

\section{INTRODUCTION}

In this paper, image based and video-based face recognition for various biometric security and recognizing specified people have been focused.

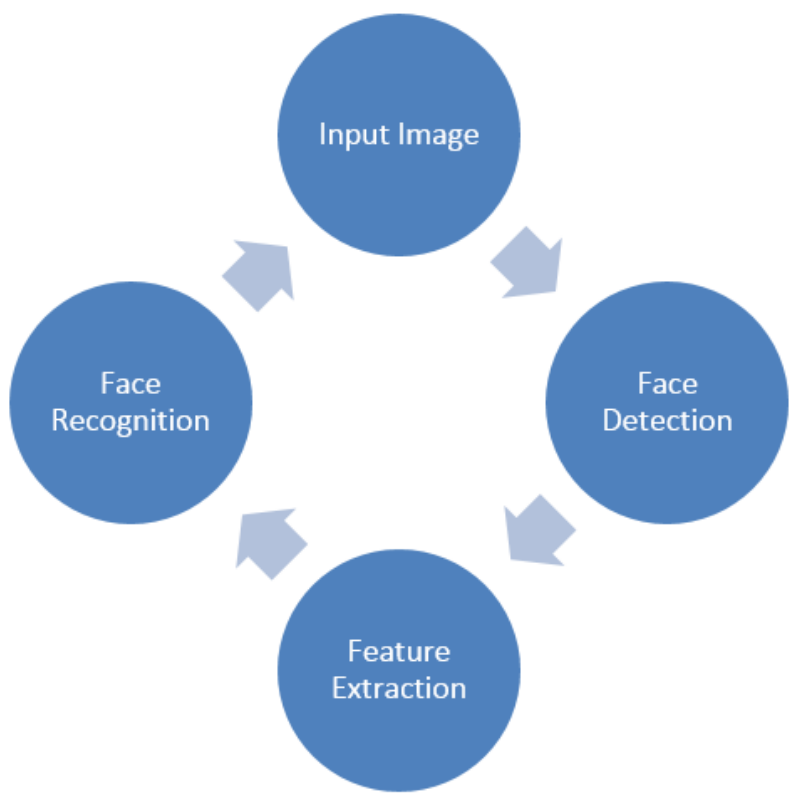

It consists of the following four steps:

$>$ Feature Extraction- Here the different patches which usually contains over 1000 pixels, are taken. Therefore, each and every patch is extracted from the given image and checked with the image saved.

Face Recognition- So, in this step the given face is identified like Facebook utilizes facial acknowledgement programming to help mechanize client labeling in photos. Each time when an individual tag in a photo the software application stores the data about the facial expressions of the given person.

$>$ Face Detection - This function determines whether the given human face appears or not and detect the face whenever required.

\section{Factor affecting face recognition technique-}

1. Face Pose- Different locations and angles of a given person's face affect the process of face recognition.

2. Face expression- Facial emotions like happy, sad, fear, anger and disgust which actually change the shape of the face affect the process. 
3. Illuminations- The lightening conditions on the given person's face at or around the background of the face affect the face recognizing process.

4. RST(rotation, scale and translation) - The rotation and the scale of the face changes the recognizing process.

\section{TECHNIQUES OF FACE RECOGNITION}

In this heading one of the oldest and most popular face recognition algorithms has been discussed which is LBPH- (Local Binary Pattern Histogram).

The steps of the given algorithm are:

\section{Major Parameters-}

a. Radius- It is used to build the circular local binary pattern and the shows radius around the central pixel, mostly it is 1 .

b. Neighbors- It is the numbers of sample point that constructs the circular local binary pattern. Mostly it is the set of 8 .

c. Grid X-It is the number of cells in the horizontal direction. More the cells, the finer will be the grid, the higher the dimension of the resulting feature vector will have. It is usually the set of 8 .

d. Grid Y- It is the number of cells in the vertical direction. It is similar to grid $\mathrm{X}$ and usually the set of 8 .

2. Training the Algorithm-Initially need to train the algorithm is there. In such process one needs to set the facial images of a given person. One also requires recognizing, and also giving one the identifying numbers. Now one can go to the next step after giving the data of the facial images.

3. Applying the LBP operation - In this the intermediate images are created that describe the original image efficiently.

Based on the given image it creates $3 \times 3$ pixels for different facial expression giving it the intensity of 0-225 which is further processed and a specific number is given for that part.

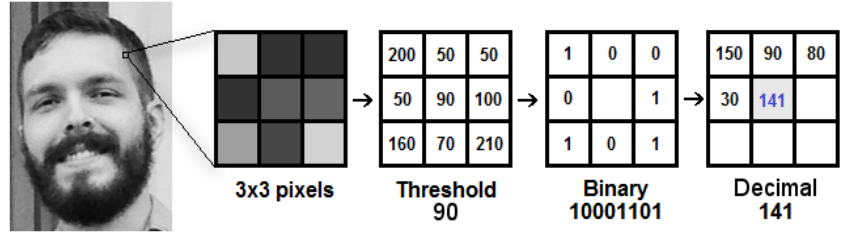

4. Extracting the histogram - Now grid $X$ and grid $Y$ parameters are used to separate the image into multiple grids as seen in the given image.

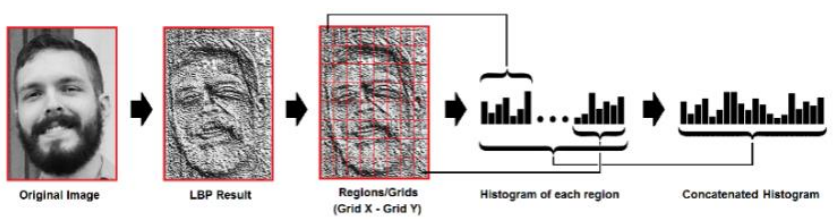

5. Performing the face recognition - Now the input image can be given and the given steps are performed:

$\checkmark$ Here, the given two histograms are compared.

$\checkmark$ In the next step the distance between the two histogram is calculated like Euclidean distance or absolute value etc. The Euclidian distance can be calculated with the help of the given formula.

$$
D=\sqrt{\sum_{i=1}^{n}\left(\text { hist } 1_{i}-\text { hist } 2_{i}\right)^{2}}
$$

By using this formula, distance will be calculated and it can be used to detect the given histogram.

\section{APPLICATIONS OF FACE RECOGNITION}

Car Security System - It is a very simple process in which the driver or the user enrolls his/her face into the car system. Therefore, whenever the user enters the vehicle again, it recognizes his face and also learns the user face time to time like giving him the permission to start the car. 
$\checkmark$ Security in Smart phones - It consists the same process as that of the security process in car, but due to various security reasons the technology behind the face recognition in the smart phone has evolved in which dot projector consisting of thousands of invisible dots is marked on the user face which creates the $3 \mathrm{D}$ face. This $3 \mathrm{D}$ face is captured with the help of flood illuminator and the infrared camera to check the user identity.

$\checkmark$ Entering and Exiting Security - In firms, hightech laboratories and military buildings, face recognition process is used similar to the smart phones security but for higher level of security the user iris is scanned thoroughly for this authentication process which is more important at this type of places.

$\checkmark$ Face recognition for potential customers- The customer face is recognized and stored in system so whenever the same customer visits the store again the system gets to know the preferences and previous order by of the customer.

$\checkmark$ Criminal Identification-Criminals can be caught by recognizing their identity with the help of high-resolution 3D camera present at the public places, near the street and other places as well. Then, the criminal face result in the system is matched with each and every person coming in the range of the camera.

\section{III.CONCLUSION}

In this paper the main concept of face recognition, factors affecting this process, technical process behind the working of face recognition and the various applications of face recognition in the field of electronic gadgets, security purposes and nabbing criminal have been enumerated.

In near future, face recognition with iris scanner and artificial intelligence for face learning is going to play a vital role for major biometric security purposes in electronic gadgets and in entry - exit systems because now a day many people can interfere with the fingerprint and can easily break the security having finger print as an authentication process. It can also be used as identifying criminal at public places with higher efficiency. This work also proposed the likelihood of correctly identifying the person of interest through the various applications.

Facial recognition is a powerful technology but it has to be used thoughtfully. On one hand, it brings numerous advantages to the companies and end-users, helps them enhance their security and track down the trespassers.

On the other hand, it may be misused for personal benefit and lead to some serious consequences. All can be done is to hope for the industry giants to make this technology more user-friendly, transparent, and secure so anyone can conveniently benefit from it.

\section{REFERENCES}

[1]. Aayushi Bansal "A study of factors Affecting face recognition." ISSN NO : 2249-7455

[2]. Ojala, Timo, Matti Pietikainen, and TopiMaenpaa. "Multiresolution gray-scale and rotation invariant texture classification with local binary patterns." IEEE Transactions on pattern analysis and machine intelligence 24.7 (2002): 971-987.

[3]. Ahonen, Timo, Abdenour Hadid, and Matti Pietikainen. "Face description with local binary patterns: Application to face recognition." IEEE transactions on pattern analysis and machine intelligence 28.12 (2006): 2037-2041.

[4]. Ahonen, Timo, Abdenour Hadid, and Matti Pietikäinen. "Face recognition with local binary patterns." Computer vision-eccv 2004 (2004): 469-481. 
[5]. LBPH Open CV : https: // docs . opencv . org / 2.4/modules/contrib/doc/facerec/facerec_tutoria 1.html\#local-binary-patterns-histograms

[6]. Local Binary Patterns : http : // www . scholarpedia .org / article / Local _ Binary _ Patterns

[7]. Kelvin salton do prado "Face Recognition: Understanding LBPH Algorithm" https://towardsdatascience.com/facerecognition-how-lbph-works-90ec258c3

\section{Authors' Profile}

Dr. Bharti Kukreja is presently working as an Associate Professor at B. S. Anangpuria Educational Institutes, Faridabad Haryana, India. She is doctorate. She has done Post Graduation in Human Resource Management and Masters in Communication Skills. Dr. Bharti Kukreja has extensive experience of working in the corporate sector and teaching at graduate as well as postgraduate level. She is playing an instrumental role helping the scholars in publishing the research papers. She has also presented numerous research papers at the international as well as national conferences and also published papers in the journals of the international repute. She keeps on delivering expert lectures on various current topics. Dr. Kukreja is the certified Language Lab trainer acknowledged by Words Worth Language Lab, an initiative of the ACTUniv Group. Dr. Kukreja has also authored a book on "Effective Technical Communication" which is being used as a course book in various universities.

The second authors namely Ritul Sharma and Shubham Sinha are the scholars of Computer Science studies in BS Anangpuria Institute of Technology \& Management, Faridabad. They have been the active part of various technical activities of the college and head the technical team as well as event management team of the college. Ritul and Shubham are the star scholars of the college and aspire to become the futuristic technocrats in the times to come. They have written various research papers for numerous journals and have been the part of various conferences of the city.

\section{Cite this article as :}

Dr. Bharti Kukreja, Ritul Kumar Sharma, Shubham Sinha, "Techniques and Applications of Face Recognition : An Overview", International Journal of Scientific Research in Science and Technology (IJSRST), Online ISSN : 2395-602X, Print ISSN : 23956011, Volume 7 Issue 1, pp. 28-31, January-February 2020. Available at doi : https://doi.org/10.32628/IJSRST20711 Journal URL : http://ijsrst.com/IJSRST20711 